\title{
Téoros
}

Revue de recherche en tourisme

\section{Entretien avec Christina Cameron, professeure, titulaire de la Chaire de recherche du Canada en patrimoine bâti - Université de Montréal (Québec, Canada)}

\section{Mathieu Dormaels}

Volume 30, numéro 2, 2011

URI : https://id.erudit.org/iderudit/1012253ar

DOI : https://doi.org/10.7202/1012253ar

Aller au sommaire du numéro

Éditeur(s)

Université du Québec à Montréal

ISSN

0712-8657 (imprimé)

1923-2705 (numérique)

Découvrir la revue

Citer ce document

Dormaels, M. (2011). Entretien avec Christina Cameron, professeure, titulaire

de la Chaire de recherche du Canada en patrimoine bâti - Université de

Montréal (Québec, Canada). Téoros, 30(2), 130-131.

https://doi.org/10.7202/1012253ar 


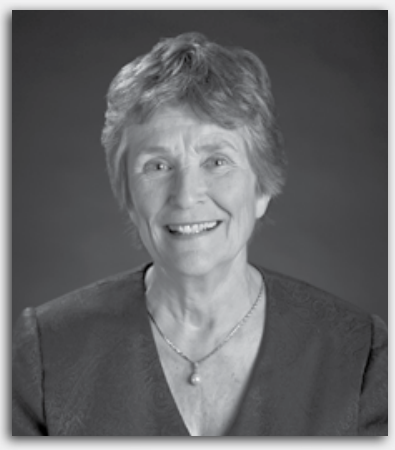

\section{Entretien}

avec Christina CAMERON, professeure, titulaire de la Chaire de recherche du Canada en patrimoine bâti - Université de Montréal (Québec, Canada)

Propos recueillis par Mathieu Dormaels

Christina Cameron est professeure à l'École d'architecture de I'Université de Montréal et titulaire de la Chaire de recherche du Canada en patrimoine bâti. Diplômée en histoire de l'art de l'Université de Toronto (B.A Hon.) et de l'Université Brown (M.A.), elle détient un doctorat en histoire de l'architecture de l'Université Laval. Elle a occupé plusieurs postes au sein de l'agence Parcs Canada où elle a notamment été directrice des lieux historiques nationaux pendant plus de 20 ans. Elle a participé puis présidé la délégation canadienne au Comité du patrimoine mondial et elle est une experte reconnue internationalement. Elle a reçu en 2006 le Prix d'excellence de la fonction publique (PSAE) du Canada pour sa carrière et en 2008 le Prix pour services insignes de la fonction publique du Canada.

\section{Comment en vient-on à travailler sur le patrimoine mondial?}

Mon parcours s'explique par ma participation, d'abord comme membre puis comme chef, dans la délégation canadienne auprès du patrimoine mondial de 1987 à 2008. Pendant un peu plus de 20 ans, en tant que haut fonctionnaire du gouvernement fédéral, je représentais le Canada, mais ce n'était pas seulement un poste de fonctionnaire pour moi. Mon intérêt personnel pour ces questions et mes intérêts de recherche liés à mon doctorat m'ont conduite à participer à des réunions d'experts et à m'engager dans la réflexion autour du patrimoine mondial et de ses enjeux. Je continue d'ailleurs à organiser des rencontres annuelles et à participer aux discussions sur la signification, la gestion et la conservation du patrimoine mondial.

\section{Sur quels sujets travaillez-vous plus particulièrement en ce moment?}

Actuellement, je travaille sur un projet qui concerne la mise en œuvre et les premières années de la Convention du patrimoine mondial. II s'agit de recueillir les « voix des pionniers », et j'ai donc interviewé une quarantaine de personnes à travers le monde qui ont participé aux débuts du patrimoine mondial ou qui en ont été des acteurs majeurs, pour comprendre ce qu'étaient leur réalité et leur vision à ces moments, comment s'est constitué le patrimoine mondial, et le mettre en relation avec ce qu'il représente aujourd'hui.

\section{Pourquoi croyez-vous qu'il soit nécessaire de revenir à cette vision des pionniers du patrimoine mondial aujourd'hui?}

Je crois que le patrimoine mondial est devenu un instrument international très fort de conservation du patrimoine puisque presque tous les pays du monde ont ratifié la Convention (NDR : 188 au 20 novembre 2011) et de nombreux sites ont été inscrits sur la Liste. Je crois que cela dépasse la vision originale de la Convention, et cela soulève des questions, surtout du point de vue des représentations du patrimoine mondial, qui est devenu une «image de marque » à succès, notamment dans le milieu touristique. II est donc important de comprendre les incidences de cette évolution dans l'équilibre entre le tourisme et la conservation. Revenir pour les examiner sur les intentions d'origine peut alimenter cette réflexion sur la situation actuelle et sur les correctifs éventuellement requis.

Le tourisme, justement, semble être devenu incontournable lorsque l'on travaille sur le patrimoine mondial.

C'est vrai aujourd'hui mais, si l'on regarde les procès-verbaux du Comité du patrimoine mondial, on voit que dans les dix premières années, le tourisme n'est mentionné que six fois, et toujours dans un contexte négatif lorsque le Comité s'est inquiété de ses impacts sur la conservation des sites. La situation est maintenant bien différente puisqu'il y a une unité au Centre du patrimoine mondial qui est dévolue au tourisme. Cependant, un récent audit sur ce sujet a mis en évidence de nombreux besoins insatisfaits et, lorsque l'on consulte les rapports périodiques ou ceux sur l'état de conservation des biens, la place du tourisme se limite encore à la dénonciation de ses impacts sur les sites, qu'ils soient culturels ou naturels. L'un des problèmes majeurs semble résider dans le fait que ce lien entre patrimoine mondial et tourisme s'avère être difficilement identifiable et gérable.

N'est-ce pas aussi du fait du tourisme que la Convention est mieux connue et qu'il y a autant de pays signataires avec autant de sites et de candidatures à l'inscription? Ce n'est pas la seule raison qui motive les pays à rechercher l'inscription de leurs sites, mais le tourisme en est une, notamment à cause de ses retombées économiques supposées. À ce titre, je crois intéressant de recommander la lecture du rapport World Heritage Status: Is there an Opportunity for Economic Gain?, établi en 2009 par les entreprises Rebanks Consulting Ltd et Trends Business Research Ltd. Cette étude relativise l'impact de l'inscription au patrimoine mondial sur l'activité touristique et ses retombées économiques qui, à moins d'une grande préparation de la communauté locale, ne semblent pas automatiquement liées à cette reconnaissance. C'est d'ailleurs un sujet important de recherches actuelles et futures que de déterminer les retombées réelles de l'inscription et son lien possible avec le tourisme. Peu d'études ont été menées sur ce sujet jusqu'à maintenant. 
II y aussi des enjeux politiques très forts aujourd'hui en ce qui a trait au patrimoine mondial, comme on l'a vu récemment avec l'entrée à l'UNESCO de la Palestine, dont la première manifestation fut l'intention de ratifier la Convention du patrimoine mondial. Dans ces enjeux politiques et économiques, quelle place peut avoir la recherche et que peuvent apporter les chercheurs? Je crois que les enjeux politiques sont liés surtout aux sites emblématiques qui permettent de renforcer l'identité nationale et qui portent aussi généralement les enjeux économiques les plus importants. Toutefois, pour une majorité de sites, la situation peut être moins politisée, ce qui ne signifie pas qu'elle soit moins compliquée aux plans de la gestion et de la protection.

En deçà des instances politiques et de leurs enjeux, la communauté universitaire a un rôle important, et unique, de se questionner sur la mise en place d'un patrimoine et d'un tourisme qui soit durable et bénéfique aux communautés locales. En effet, la bonne gestion du patrimoine repose finalement sur les communautés locales qui doivent, au bout du compte, être mieux outillées pour faire face à des situations politiques ou économiques problématiques, sans préjudices aux sites ou aux modes de vie de ces communautés. Sur tous ces sujets, la recherche a beaucoup à apporter parce que ce ne sont pas des questions qui peuvent être abordées lors des discussions pour l'inscription ou lors de l'examen de l'état de conservation des sites. Ce doit être une réflexion plus profonde, qui peut être menée par des chercheurs ayant suffisamment de temps et de recul par rapport aux États et à l'institution internationale.

\section{La Convention va fêter ses 40 ans l'année prochaine et, selon vous, vers quelle situation le patrimoine mondial est-il en train d'évoluer?}

C'est difficile à dire, mais je crois que l'on va connaître bientôt une période de réflexion autour du fonctionnement et de la gestion de la Liste du patrimoine mondial. En effet, l'activité commence à être très importante avec, par exemple, des réunions du Comité qui était de quatre jours au départ alors que l'on prévoit déjà douze ou treize jours pour la prochaine réunion en 2012. Le système devient donc de plus en plus important et difficile à administrer. II faudra nécessairement réfléchir, non seulement aux problèmes de gestion de la Liste, aux éventuelles nouvelles inscriptions, mais aussi aux questions de conservation des sites.

\footnotetext{
II n'y a pas que l'administration du patrimoine mondial qui change, les visiteurs évoluent aussi n'est-ce pas? Selon le département régional du tourisme à Siem Reap, au Cambodge, le nombre de visiteurs étrangers en 2011 aux temples d'Angkor Vat' a augmenté de $23 \%$ par rapport à l'an dernier. Ce qui est plus remarquable, c'est que ces 1,6 millions de touristes proviennent surtout des autres pays de l'Asie. Parmi les quatre principaux pays d'origine de ces visiteurs se trouvent la Corée du Sud (265 000, en augmentation de $31 \%$ ), le Viet Nam (251 400, en augmentation de $45 \%$ ), le Japon (120 000, en augmentation de $5 \%$ ) et la Chine (119 900, en augmentation de $81 \%$ ). On attribue notamment cette tendance à l'amélioration des réseaux de transport aérien entre Siem Reap et les pays de la région. Ces changements ont des implications dans de nombreux aspects de la protection et de la mise en valeur des sites, et il faut aussi les prendre en considération dans les réflexions qui sont entreprises.
}

\section{Et les chercheurs auront-ils un rôle à jouer dans cette réflexion?}

Oui, certainement, car les États agissent en fonction de leurs intérêts politiques nationaux tandis que les chercheurs ont la possibilité de prendre de la distance et d'examiner le système dans son ensemble, au-delà des points de vue nationaux.

C'est ce qui leur donne la possibilité de mettre en évidence les tendances et de contribuer à la discussion entre les États parties. Je vois d'ailleurs de plus en plus d'universitaires s'organiser en réseau, en dehors du système intergouvernemental, et qui s'intéressent aux questions soulevées par les enjeux actuels du patrimoine mondial.

\section{$Y$ a-t-il d'autres sujets qui, selon vous, pourraient faire l'objet de recherche dans les prochaines années?}

En ce qui concerne les relations entre le tourisme et le patrimoine mondial, il me paraît important de porter plus d'attention à ce qui se passe en Asie. C'est une partie du monde où l'on compte de plus en plus de sites et où ceux-ci connaissent un tourisme très important. La fréquentation de la Grande Muraille de Chine a reçu plus de 10 millions de visiteurs l'an dernier, soit un tiers de la population du Canada et plus que la population totale du Québec ! II devient donc urgent de se poser les bonnes questions sur la gestion et la conservation de ces sites, et pour cela il faut les étudier pour déterminer quelles sont les conséquences de cette fréquentation et comment faire pour trouver un équilibre entre la préservation, le tourisme et le bénéfice des communautés locales.

Note

1 À propos de ce site, voir l'article d'analyse publié par Olivier Lazzarotti (2001) «Angkor: Faut-il réveiller la Belle au bois dormant?», Téoros, vol. 20, n³, p. 54-58. 